\title{
Endothelium-Mediated Hepatocyte Recruitment in the Establishment of Liver-like Tissue In Vitro
}

\author{
YAAKOV NAHMIAS, ${ }^{1}$ ROBERT E. SCHWARTZ, ${ }^{2}$ WEI-SHOU HU, ${ }^{3}$ \\ CATHERINE M. VERFAILLIE, ${ }^{2}$ and DAVID J. ODDE ${ }^{1}$
}

\begin{abstract}
A major goal of liver tissue engineering is to understand how the constituent cell types interact to achieve liver-specific structure and function. Here we show that hepatocytes migrate toward and adhere to endothelial vascular structures formed on Matrigel in vitro, and that hepatocyte recruitment is dependent on endothelium-derived hepatocyte growth factor. The hepatocyte-decorated endothelial vascular structures resemble in vivo sinusoids containing plate-like structures, bile canaliculi, and a lumen. The sinusoid-like structures retained cytochrome P450 expression and activity, in addition to stable albumin expression and secretion rate for over 2 months in vitro. The stability of the sinusoid-like structures was dependent on the presence of vimentin-positive fibroblasts in culture. The sinusoid-like structures formed by hepatocytes and pure populations of endothelial cells collapsed after 10 days in culture. In contrast, culture of hepatocytes with fibroblast-contaminated human dermal microvascular endothelial cells or a combination of human umbilical vein endothelial cells and normal human dermal fibroblasts resulted in stable sinusoid-like structures surrounded by a fibroblastic capsule that maintained liver specific functions for several months in vitro. These results demonstrate that specification of endothelial cell position ultimately determines hepatocyte position in vitro, suggesting that similar interactions might occur in vivo. The novelty of the culture's sinusoid-like organization and long-term function suggest a new model for the study of liver toxicity, ischemia/reperfusion injury, and fibrosis.
\end{abstract}

\section{INTRODUCTION}

$\mathbf{T}$ The LIVER's Elaborate ARCHITECTURE and heterotypic cell interactions are required for the phenotypic stability of the parenchymal cells as well as for proper liver function. ${ }^{1,2}$ Loss of organization and aberrant cell interaction lead to liver failure. Although hepatocyte cell culture techniques have developed to the point where hepatocyte function and limited proliferation are possible, the inability to recreate functional liver-like tissue, such as sinusoids, in vitro ${ }^{3}$ limits our capability to study liver development, ${ }^{4}$ regeneration, ${ }^{5}$ ischemia/reperfusion injury, ${ }^{6}$ toxicity, ${ }^{7}$ fibrosis, ${ }^{8}$ and viral infection. ${ }^{9}$

The liver's unique organization arises during development when cords of hepatocytes emerge from the hepatic diverticulum, migrate toward, and associate with vascular structures of the septum transversum, forming the hepatic sinusoids. ${ }^{4,10}$ The most extensive hepatic outgrowth occurs only after endothelial cells have assembled into vascular structures. ${ }^{4}$ Similarly, after liver regeneration,

\footnotetext{
${ }^{1}$ Department of Biomedical Engineering, University of Minnesota, Minneapolis, Minnesota.

${ }^{2}$ Stem Cell Institute, University of Minnesota, Minneapolis, Minnesota.

${ }^{3}$ Department of Chemical Engineering, Materials Science, University of Minneapolis, Minneapolis, Minnesota.
} 
hepatocytes clusters are invaded by matrix depositing stellate cells, followed by growing endothelial cells that form capillaries. ${ }^{11}$ Hepatocytes then associate with the newly formed capillaries restoring normal liver architecture. The interactions between hepatocytes and endothelial vessels leading to liver morphogenesis have yet to be recaptured in vitro.

Several groups have reported the spontaneous formation of hepatic organoids by co-culturing hepatocytes with the nonparenchymal fraction of the liver. Small hepatocytes, characterized by Mitaka et al., proliferate to form colonies supported by epithelial cells and fibroblasts with an extensive bile canaliculi network and differentiated function. ${ }^{12}$ Hepatocyte cords emanated from the colony in response to Matrigel. ${ }^{13}$ Likewise, Michalopoulos et al. found that hepatic plates are formed when parenchymal and nonparenchymal cells are cultured on carrier beads in Matrigel. ${ }^{14}$ In another study, the same group demonstrated that parenchymal and nonparenchymal cells self-organize in roller bottles to form simple epithelial structures consisting of a superficial layer of biliary epithelial cells, a middle layer of hepatocytes and connective tissue, and an inner layer of endothelial cells. ${ }^{15}$ Several other groups have shown that hepatic function is supported by close contact of either patterned fibroblasts ${ }^{16}$ or overlaid endothelial cells. ${ }^{17}$

Although aspects of liver function have been preserved by various methods in vitro, the actual organization of the liver sinusoid (endothelial vessels coated with hepatic tissue) has never been recreated. The organization and function of the liver sinusoid has been shown to be important for the study of ischemia/reperfusion injury, ${ }^{6}$ drug-induced toxicity, ${ }^{4,18}$ fibrosis, ${ }^{8,9}$ and cirrhosis. ${ }^{19}$ One approach to recreate a sinusoid-like element in vitro is to co-culture hepatocytes on endothelial vascular structures formed on Matrigel, a commonly used angiogenesis assay. ${ }^{20}$ This approach would also allow one to directly observe the dynamics (cellular interaction, association) of the system for the study of organogenesis, regeneration, differentiation, infection, and toxicity.

Here, we demonstrate the ability of endothelial vascular tubes to direct and support liver sinusoid formation in an in vitro Matrigel assay. The sinusoid-like structures retained stable cytochrome $\mathrm{P} 450$ gene expression and activity, significantly greater than that of traditional monolayer culture systems. In addition, the structures retained a stable albumin gene expression and secretion rate for more than 2 months in vitro. Our ability to establish liverlike organization and function for months in vitro offers the possibility to study the heterotypic cell-cell interactions shown to be important for proper liver function, under carefully controlled conditions, and suggests a new method for the study of liver toxicity, ischemia/reperfusion injury, and fibrosis. ${ }^{3}$

\section{MATERIALS AND METHODS}

\section{Materials}

Williams medium E, M199 basal medium, penicillin/streptomycin, L-glutamine, sodium pyruvate, and insulin were purchased from Invitrogen Life Technologies (Carlsbad, CA). Glycine, dexamethasone, and heparin were obtained from Sigma-Aldrich (St. Louis, MO). Epidermal growth factor (EGF) and growth factor-reduced phenol red-free Matrigel was obtained from Fisher Scientific (Pittsburgh, PA), endothelial cell growth factor (ECGF) from Roche Applied Science (Indianapolis, IN), recombinant human vascular endothelial growth factor (VEGF) and hepatocyte growth factor (HGF) from R\&D Systems Inc. (Minneapolis, MN), fetal bovine serum heat-inactivated from Hyclone Laboratories (Logan, UT), and EndoGro from Vec Technologies (Rensselaer, NY). Immunofluorescence grade paraformaldehyde was purchased from Electron Microscope Sciences (Hatfield, PA). Normal donkey serum and secondary $\mathrm{F}\left(\mathrm{ab}^{\prime}\right) 2$ antibody fragments, ML grade were obtained from Jackson Immunoresearch (Bar Harbor, ME). DiIAc-LDL was purchased from Biomedical Technologies (Stoughton, MA). Mouse anti-rat CK18, 1:50 dilution was from Sigma Aldrich (St. Louis, MO). Mouse antihuman CD31, 1:10 dilution was from Becton-Dickinson (San Diego, CA). Mouse anti-human desmin II, 1:12 dilution was from ICN (Costa Mesa, CA). Rabbit anti-rat albumin, 1:500 dilution was from ICN. Neutralizing antibodies against HGF and stromal cell-derived factor 1 (SDF-1) were purchased from R\&D Systems Inc. (Minneapolis, MN).

\section{Hepatocyte isolation and culture}

Hepatocytes were harvested from 4- to 6-week-old male Sprague-Dawley rats weighing $175-275 \mathrm{~g}$ by a two-step in situ collagenase perfusion technique. ${ }^{21}$ Hepatocyte viability after the harvest was more than $80 \%$ based on trypan blue exclusion. All animals were treated according to protocols approved by the animal care institutional review board. Hepatocyte purity was greater than $95 \%$.

Human hepatocytes were harvested from 5 to $60 \mathrm{~g}$ resections from 19- to 56-year-old human patients. Briefly, liver resections were washed with a lactated Ringer solution containing heparin and kept at $4^{\circ} \mathrm{C}$. Small $1-2-\mathrm{cm}$ square pieces were sectioned and perfused with a modified two-step in situ collagenase perfusion technique. ${ }^{21}$ Hepatocyte viability after harvest was assessed by trypan blue exclusion.

\section{Endothelial cell isolation and culture}

Rat liver sinusoidal endothelial cells (LSEC) were isolated from the nonparenchymal fraction of the liver us- 
ing a two-step Percoll gradient (25/50\%) separation following the procedure of Zhang et $_{\text {al. }}{ }^{22}$ LSEC were seeded directly on Matrigel for co-culture studies.

Human umbilical-vein endothelial cells (HUVEC) and human microvessel endothelial cells (MVEC) were a kind gift from Dr. Robert Hebbel, University of Minnesota. ${ }^{23}$ Cells were cultured on either $0.2 \%$ (HUVEC) or 1\% (MVEC) gelatin-coated T-75 flasks and split 1:3 when confluent. Endothelial culture medium consisted of M199 basal medium, 15\% fetal bovine serum (FBS), EndoGro $(40-50 \mathrm{mg} / \mathrm{mL})$, L-glutamine $(0.7 \mathrm{mg} / \mathrm{mL})$, heparin $(10 \mathrm{U} / \mathrm{mL})$, sodium pyruvate $(30 \mu \mathrm{g} / \mathrm{mL})$, and penicillin/streptomycin $(100 \mathrm{U} / \mathrm{mL})$. Cell medium was changed every 2 days.

Normal human dermal fibroblasts (NHDF) were a kind gift from Dr. Robert Tranquillo, University of Minnesota. Cells were cultured on uncoated T-75 tissue culture flasks and split 1:3 when confluent. NHDF culture medium consisted of Dulbecco's Modified Eagle Medium basal medium, $10 \%$ calf serum, glucose $(4.5 \mathrm{mg} / \mathrm{mL})$, sodium bicarbonate $(1.5 \mathrm{mg} / \mathrm{mL})$, L-glutamine $(4 \mathrm{mM})$, sodium pyruvate $(0.110 \mathrm{mg} / \mathrm{mL})$, and $1 \%$ penicillin, streptomycin, and amphotericin B. Cells were maintained in a $5 \% \mathrm{CO}_{2}$ humidified incubator at $37^{\circ} \mathrm{C}$. Cell medium was changed every 2 to 3 days.

\section{Co-cultures of cells on Matrigel slides}

Matrigel aliquots $(200 \mu \mathrm{L})$ were thawed overnight at $4^{\circ} \mathrm{C}$, layered on ice-cold glass chamber slides $(0.5-\mathrm{mm}$ thickness, $4 \mathrm{~cm}^{2}$ ), and incubated at $37^{\circ} \mathrm{C}$ for $30 \mathrm{~min}$ to allow the gel to form. HUVECs, MVECs at an early passage number $(<4)$, or fresh LSECs were layered on top of the gel at a density of $50,000 \mathrm{cells} / \mathrm{cm}^{2}$ in $1 \mathrm{~mL}$ of endothelial cell medium. Freshly isolated hepatocytes were added at a density of 10,000 cells $/ \mathrm{cm}^{2}$ in an additional milliliter of coculture medium. Co-culture medium consisted of Williams Medium E, HEPES, pen/strep (100 U/mL), and insulin (20 $\mathrm{mU} / \mathrm{mL}$ ), which was stored for up to 4 weeks in $4^{\circ} \mathrm{C}$. To use in culture experiments, the medium was supplemented once a week with $2 \%$ FBS, L-glutamine $(0.3 \mathrm{mg} / \mathrm{mL})$, dexamethasone $\left(10^{-8} \mathrm{M}\right)$, ECGF $(20 \mu \mathrm{g} / \mathrm{mL})$, heparin $(10$ $\mathrm{U} / \mathrm{mL})$, EGF, and VEGF $(10 \mathrm{ng} / \mathrm{mL})$. Cells were allowed to attach overnight, at which time the medium was replaced with $1 \mathrm{~mL}$ of co-culture medium. Co-culture medium was replaced every 2 days and the spent media was stored at $-20^{\circ} \mathrm{C}$ for albumin analysis.

\section{Hepatocyte-endothelium migration assays}

Endothelial-hepatocyte chamber slides were prepared as described above. Endothelial cells were seeded at 50,000 cells $/ \mathrm{cm}^{2}$ and allowed to form networks overnight. One hour before the addition of hepatocytes, the cells were incubated with a neutralizing antibody against
HGF (10 $\mu \mathrm{g} / \mathrm{mL})$, SDF-1 (5 $\mu \mathrm{g} / \mathrm{mL})$, both, or none of the antibodies. Hepatocytes were then seeded at 10,000 cells $/ \mathrm{cm}^{2}$ and allowed $30 \mathrm{~min}$ to adhere. Two fields of view in every chamber were imaged every $30,90,180$, 510,930 , and $1680 \mathrm{~min}$ on a tissue culture microscope (Nikon, Eclipse TS100). The images were recorded by a CCD camera (Sony XC-75), captured on computer using a frame grabber (LG-3, Scion Imaging), and digitally analyzed in Scion Image. Only cells that were within 150$\mu \mathrm{m}$ distance (hepatocyte spheroid size) to the nearest vascular structure were followed. Cells already in contact with the structures were ignored. A successful migration was defined by a cell moving from its starting location and making contact with the endothelial structures within the specified time.

\section{Western blot analysis}

Western blot analyses were performed as previously described. ${ }^{24}$ The antibody to HGF was from R\&D Systems Inc. (Minneapolis, MN). Secondary antibodies were horseradish peroxidase linked donkey anti-goat (Amersham Life Sciences Inc., Arlington Heights, IL). Enhanced chemiluminescence was performed according to the manufacturer's instructions (Amersham Life Sciences Inc.).

\section{Total RNA isolation and PCR}

RNA was extracted from $3 \times 10^{5}$ HUVEC plated onto Matrigel for 2 days, $3 \times 10^{5}$ rat hepatocytes, or $10 \mathrm{mg}$ of freshly isolated human liver tissue. The mRNA was reverse transcribed and cDNA was amplified as follows: 40 cycles of a two-step polymerase chain reaction (PCR) $\left(95^{\circ} \mathrm{C}\right.$ for $15 \mathrm{~s}, 60^{\circ} \mathrm{C}$ for $\left.60 \mathrm{~s}\right)$ after initial denaturation $\left(95^{\circ} \mathrm{C}\right.$ for $10 \mathrm{~min}$ ) with $2 \mu \mathrm{L}$ DNA solution, $1 \mathrm{x}$ TaqMan universal mix PCR reaction buffer using a MJ Research thermal cycler (MJ Research, Inc., Reno, NV). Primer sequences are available by request.

\section{Transmission electron microscopy}

The cells were fixed in $2.5 \%$ glutaraldehyde in $0.1 \mathrm{M}$ cacodylate buffer $(\mathrm{pH}$ 7.4) containing sucrose at room temperature for 30 min, postfixed in $2 \%$ osmium tetroxide in the buffer, and embedded in situ in Epon 812. Semithin and ultrathin sections were cut on Reichert Ultracut E and RMC MT-7000 ultramicrotomes. The semithin sections were stained with toluidine blue and examined with a light microscope. The adjacent thin sections were stained with uranyl acetate followed by lead citrate and examined at $60 \mathrm{KV}$ with a JEM transmission electron microscope (JEOL, Tokyo, Japan).

\section{Immunofluorescence microscopy}

Chamber slide samples were washed three times with PBS, and fixed in 4\% EM-grade paraformaldehyde for 

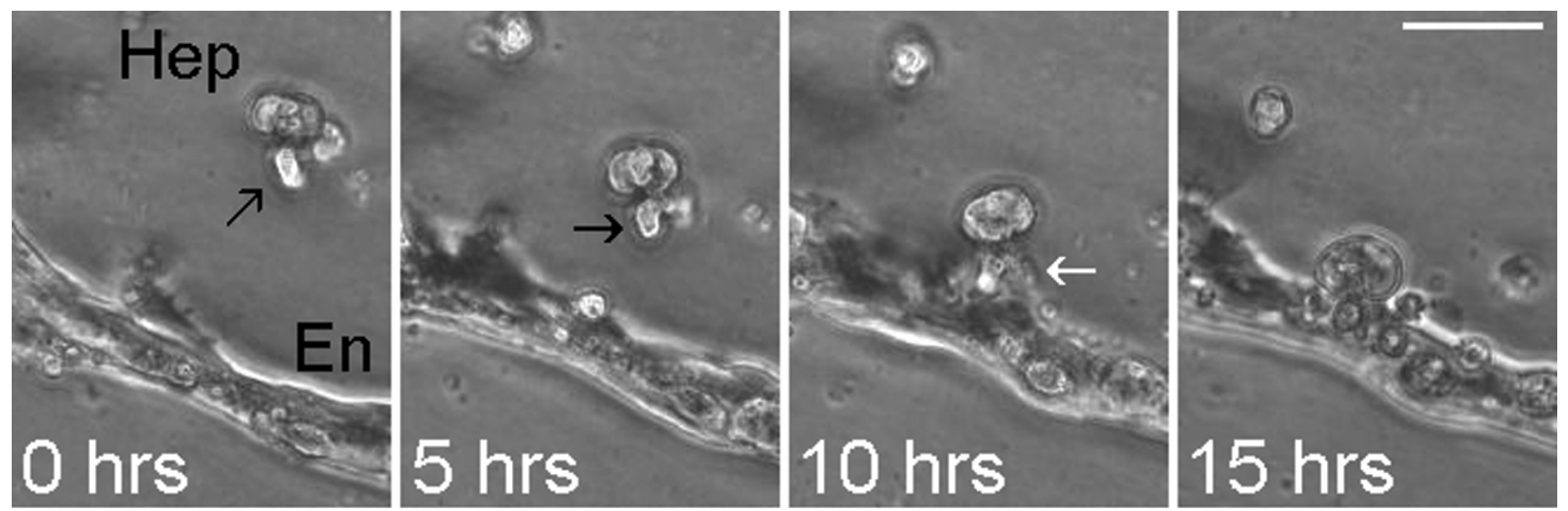

FIG. 1. Endothelium-mediated recruitment of hepatocytes. Time-lapse recording of hepatocyte (Hep) migration toward endothelial tube-like structures (En), at 5-h intervals. Note the pseudopod structure that extends in the direction of migration (black arrow). In certain instances, endothelial processes extended to grab the approaching hepatocyte (white arrow). Bar $=50 \mu \mathrm{m}$.

$10 \mathrm{~min}$ at room temperature. Slides were then washed with PBS and incubated in $100 \mathrm{mM}$ glycine for $15 \mathrm{~min}$ to saturate reactive groups. Samples were permeabilized for 15 min with $0.1 \%$ Triton X-100, blocked for $30 \mathrm{~min}$ with $1 \%$ bovine serum albumin, $5 \%$ Donkey Serum at room temperature followed by staining with primary antibodies overnight at $4^{\circ} \mathrm{C}$. After additional washes with PBS, samples were stained with fluorescently tagged secondary antibodies for $45 \mathrm{~min}$ at room temperature.

\section{Assessment of PROD activity}

To probe for the rat cytochrome $\mathrm{P} 450$ 2B1/2 (CYP2B1/2) activity in hepatocyte co-cultures, we used the pentoxyresofurin-O-depentylation (PROD) reaction. Nonfluorescent pentoxyresorufin is a specific substrate for rat CYP2B $1 / 2 .{ }^{25}$ The product of the PROD reaction is fluorescent resorufin, which can be easily detected in the cells.

Briefly, culture medium was replaced with $1 \mathrm{~mL}$ of prewarmed incubation buffer and incubated for $15 \mathrm{~min}$ in $5 \% \mathrm{CO}_{2}, 37^{\circ} \mathrm{C}$. The incubation buffer was incomplete Williams' E medium supplemented with $2 \mathrm{mM}$ probenicid (Sigma), an inhibitor of glucuronidation, and $25 \mu \mathrm{M}$ dicumarol (Sigma), a specific inhibitor of DT-diaphorase. Dicumarol inhibits further metabolism of resorufin ${ }^{26}$ to nonfluorescent metabolites. The stock solutions of $0.2 \mathrm{M}$ probenicid and $10 \mathrm{mM}$ dicumarol were prepared in 0.5 $\mathrm{N} \mathrm{NaOH}$, and $\mathrm{pH}$ adjusted to 7.2 with 1.0 M HEPES. The PROD reaction was initiated by the addition of 20 $\mu \mathrm{M}$ pentoxyresorufin (Molecular Probes). The stock solution of pentoxyresorufin was at $1 \mathrm{mM}$ in DMSO.

\section{RESULTS}

\section{Early co-culture of endothelial cells and hepatocytes}

Matrigel is a commonly used angiogenesis assay ${ }^{27}$ and has been shown to preserve hepatocyte function in

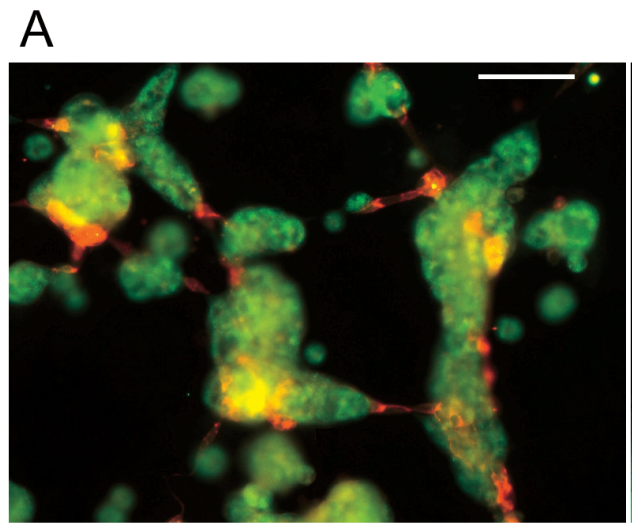

B

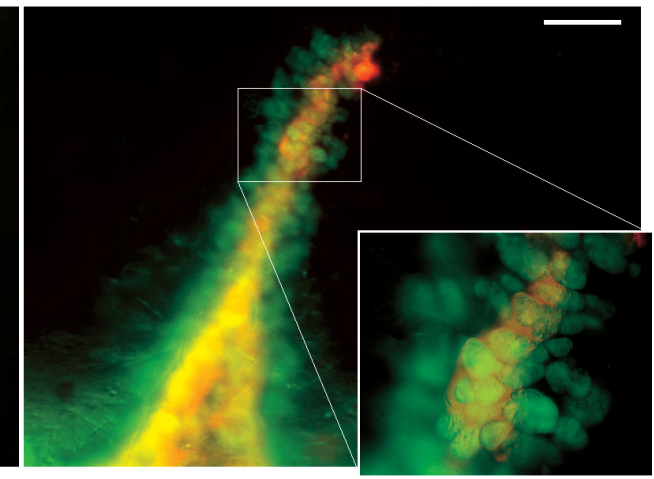

FIG. 2. Sinusoid-like structure formed in vitro. (A) Double immunofluorescence staining for endothelial cells (CD31, red) and hepatocytes (CK-18, green) in hepatocyte-HUVEC co-cultures at day 2 in culture. Bar $=100 \mu \mathrm{m}$. (B) Hepatocytes decorating MVEC tube at day 4. Bar $=200 \mu \mathrm{m}$. 
vitro. ${ }^{28}$ In order to study the interaction between endothelial cells and hepatocytes, we seeded either MVECs, HUVECs, or rat LSECs on Matrigel. The endothelial cells rapidly formed tube-like structures. Freshly isolated rat $(n=9)$ or human $(n=2)$ hepatocytes were then added at low density $\left(10,000\right.$ cells $\left./ \mathrm{cm}^{2}\right)$ to cultures with pre-established vascular tubes. The randomly seeded hepatocytes then migrated toward the vascular tubes (Fig. 1) and remained adherent to them (Fig. 2). No differences were seen between the three types of endothelium or across species. We never observed hepatocyte migration away from the nearest tube-like structure. When hepatocytes were plated at low density in the same base medium (which lacks HGF) but in the absence of endothelium, they failed to aggregate ( $n=$ 12, Fig. 3). Hepatocytes seeded at a higher density $\left(>40,000\right.$ cells $\left./ \mathrm{cm}^{2}\right)$ or in the presence of HGF aggregated normally.

\section{$H G F$ recruits hepatocytes to endothelial cells}

HGF is a key hepatocyte mitogen and motogen produced by nonparenchymal cells of the liver. To detect whether endothelial-secreted HGF plays a role in the migration of hepatocytes, we analyzed the cultures for the presence of HGF. High levels of HGF mRNA $(n=5)$ and protein $(n=4)$ were detected in both HUVEC and MVEC cell lysates after culture on Matrigel (Fig. 4A and B). In addition, intracellular HGF was also detected in HUVEC vascular structures by immunofluorescence (Fig. 5A and B). Finally, the addition of neutralizing antibodies against HGF $(10 \mu \mathrm{g} / \mathrm{mL})$ significantly decreased

\section{Hepatocyte}

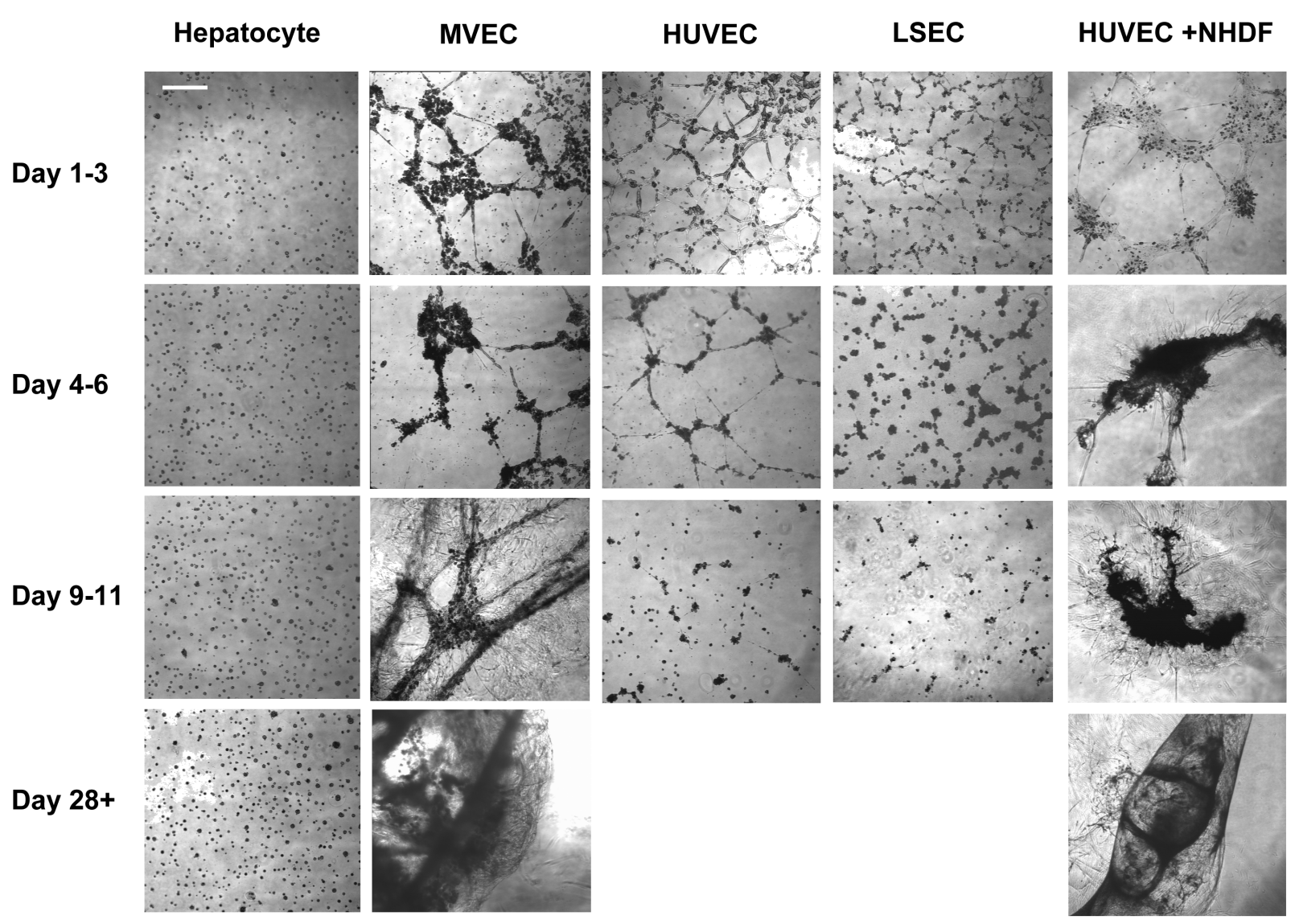

FIG. 3. Morphological changes in co-cultures of hepatocytes and endothelial cells. Hepatocytes cultured alone at low densities $\left(10,000 \mathrm{cell} / \mathrm{cm}^{2}\right)$ on Matrigel do not aggregate even after 28 days in culture. Culture medium contained $10 \mathrm{ng} / \mathrm{mL}$ EGF but no HGF. Three different types of endothelial cells show similar interaction with hepatocytes during short-term culture (0-6 days). Hepatocytes selectively migrate toward and adhere to endothelial tube-like structures, creating sinusoid-like structures. By day 10, endothelial networks of HUVEC and LSEC collapse, causing the death of associated hepatocytes as well. In contrast, the cellular networks created by either MVEC or HUVEC-NHDF co-culture remain. An outgrowth of fibroblast-like cells quickly emerges in MVEC or HUVECNHDF culture, creating a mat on which the structures sit, while simultaneously degrading the gel. A few weeks later the sinusoidlike structures are encased in a fibroblastic capsule and the capsule detaches from the dish. Bar $=500 \mu \mathrm{m}$. 


\section{Matrigel HGF HUVEC HEP MVEC}

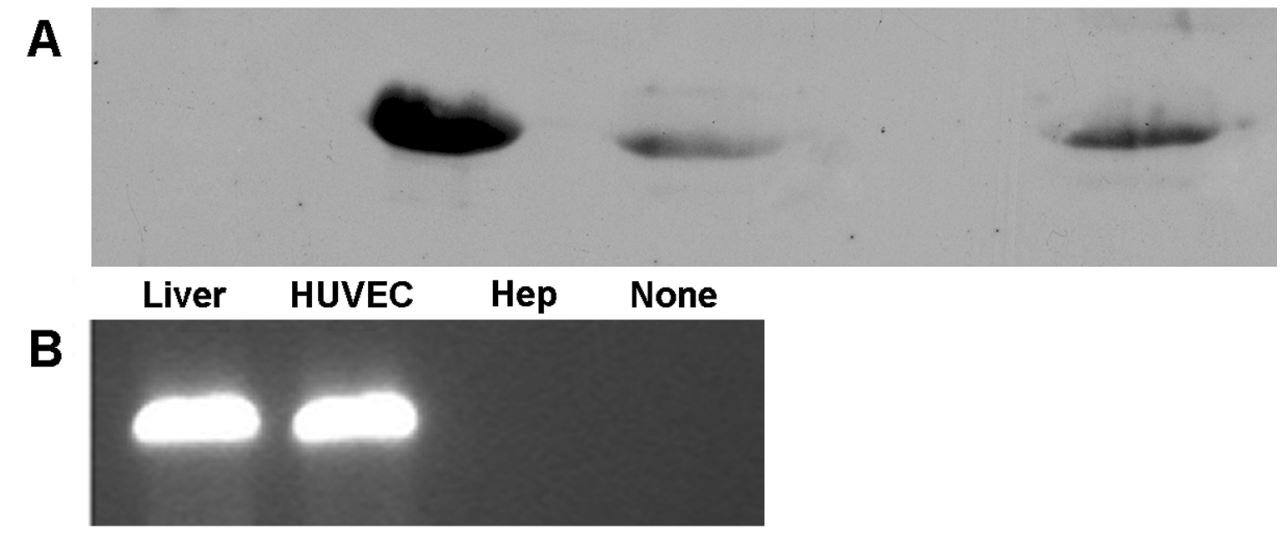

FIG. 4. Detection of HGF protein and mRNA in endothelial cells. (A) Western blot analysis of HGF from HUVEC and MVEC. A band of $85 \mathrm{kD}$ is found that corresponds to native HGF. Ten micrograms of each protein sample was run for all lanes except for HGF, where $500 \mathrm{ng}$ of protein was run in each lane. (B) PCR analysis of HGF expression. Briefly, after 2 days of culture on Matrigel, HUVEC were lysed, and their RNA was isolated and treated with DNAse. The product was then reverse transcribed into DNA and analyzed for HGF expression by PCR analysis. Lane 1 is HGF expression by human liver tissue, lane 2 is HUVEC cultured on Matrigel, lane 3 is rat hepatocytes, and lane 4 is a no-template control. Controls lacking reverse-transcriptase were negative for all samples.

hepatocyte migration toward HUVEC-derived vascular tubes (Fig. 5C). SDF-1 is also a potent chemoattractant. Mice lacking SDF-1 or its receptor, CXCR4, have impaired vascularization of the gastrointestinal tract. ${ }^{29}$ In addition, studies have suggested that engraftment of hepatocyte progenitor cells in the liver depends on SDF$1 .{ }^{30,31}$ In contrast to the activity of HGF, the addition of neutralizing antibodies against SDF-1 $(5 \mu \mathrm{g} / \mathrm{mL})$ did not have an effect on hepatocyte migration under the same conditions (Fig. 5C). Together, these results indicate that endothelium-secreted HGF directs hepatocyte migration toward the vascular tubes on Matrigel.
It is important to note that our migration assay did not account for hepatocytes in contact with the endothelial vascular structures that rapidly adhered to the vessels. In addition, our media contained low concentrations of growth factors, and did not contain HGF, accounting for the low percentage of migrating hepatocytes.

\section{Long-term culture of sinusoid structures}

Long-term stability of the sinusoid-like structures was dependent on the presence of fibroblasts in the culture. Sinusoid-like structures created by HUVECs and LSECs
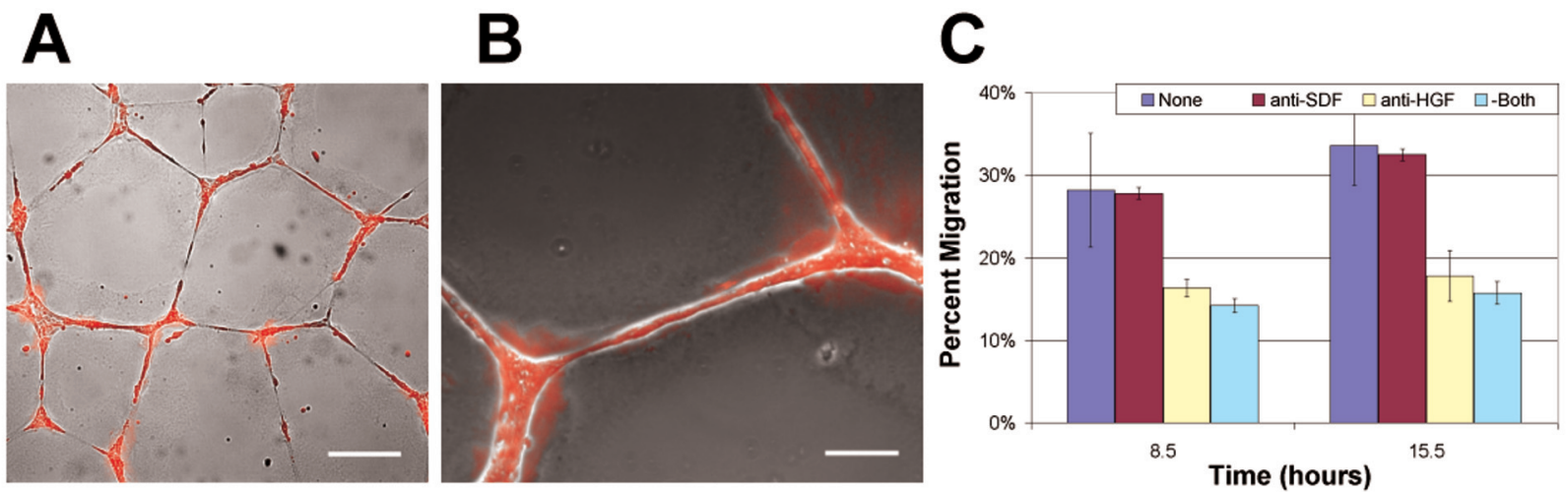

FIG. 5. HGF-dependent hepatocyte recruitment by endothelial vascular structures. (A) Intracellular HGF staining in HUVEC vascular tubes $24 \mathrm{~h}$ after seeding. Bar $=500 \mu \mathrm{m}$. (B) A higher magnification image of HGF staining in HUVEC vascular tubes and bound to the surrounding gel at day 3 . Bar $=200 \mu \mathrm{m}$. (C) The percent of hepatocytes that migrated (mean \pm s.d.) is significantly inhibited by the addition of anti-HGF antibodies ( $p=5.4 \times 10^{-5}, 1500$ cells, $N=3$ experiments), but not by addition of anti-SDF-1 antibodies $(p=0.269)$. 

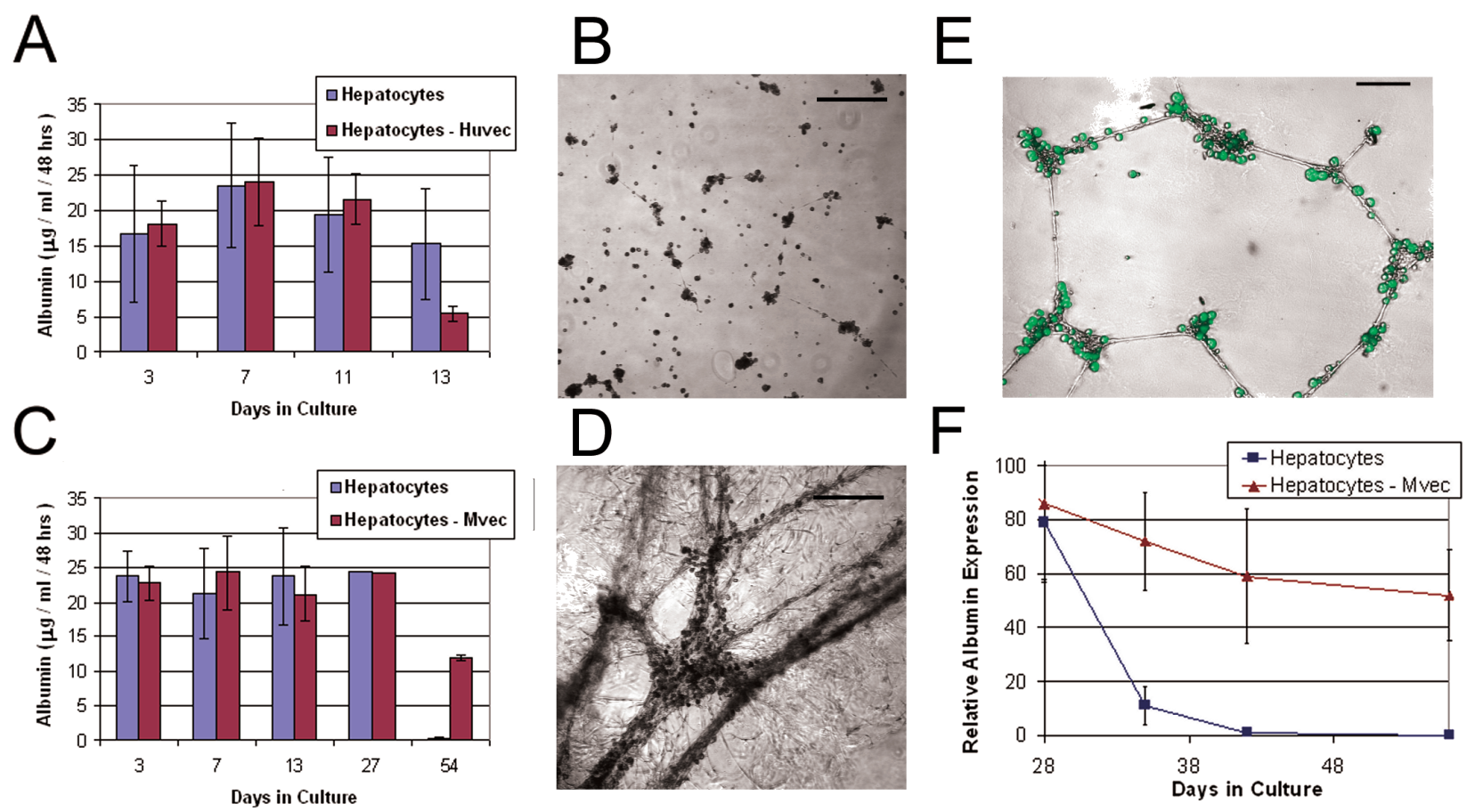

FIG. 6. Albumin expression and secretion by sinusoid-like structures. (A) Albumin secretion in hepatocyte/endothelial co-cultures is comparable to that of freshly isolated hepatocytes cultured on Matrigel $(40,000$ cells $/ \mathrm{mL})$, but ultimately decreases after 2 weeks. (B) HUVEC structures on Matrigel are transient and collapse by day 10 of culture, leading to a significant loss of albumin secretion compared to isolated hepatocyte cultures. Bar $=500 \mu \mathrm{m}$. (C, D) MVEC sinusoid-like structures are stable and maintain albumin secretion for more than 1 month in culture. Bar $=500 \mu \mathrm{m}$. (E) Intracellular albumin staining in hepatocytes decorating HUVEC vascular tubes. Bar $=200 \mu \mathrm{m}$. (F) Quantitative reverse transcriptase (RT)-PCR confirms that hepatocyte-MVEC co-culture maintains albumin expression during long-term culture, whereas a pure hepatocyte culture loses expression after 28 days on Matrigel. Controls lacking RT were negative for all samples and are not shown. Values are means and standard deviations of three experiments.

were transient, collapsing after a week in culture with an associated loss in albumin secretion and cell death (Fig. $6 \mathrm{~A}$ and $\mathrm{B})$. In contrast, sinusoid-like structures created by MVECs showed stable albumin gene expression and secretion up to 2 months in culture (Fig. 6C, D, and F). Hepatocytes in single culture lost albumin expression and secretion after a month in culture (Fig. 6c and f). After a week in culture, an outgrowth of fibroblast-like cells emerged from the MVEC network (Fig. 7A and B). Immunofluorescent staining of the fibroblast-like cells demonstrated that they were CD31 and CK18 negative, while vimentin and calponin positive, suggesting a pericyte ori-
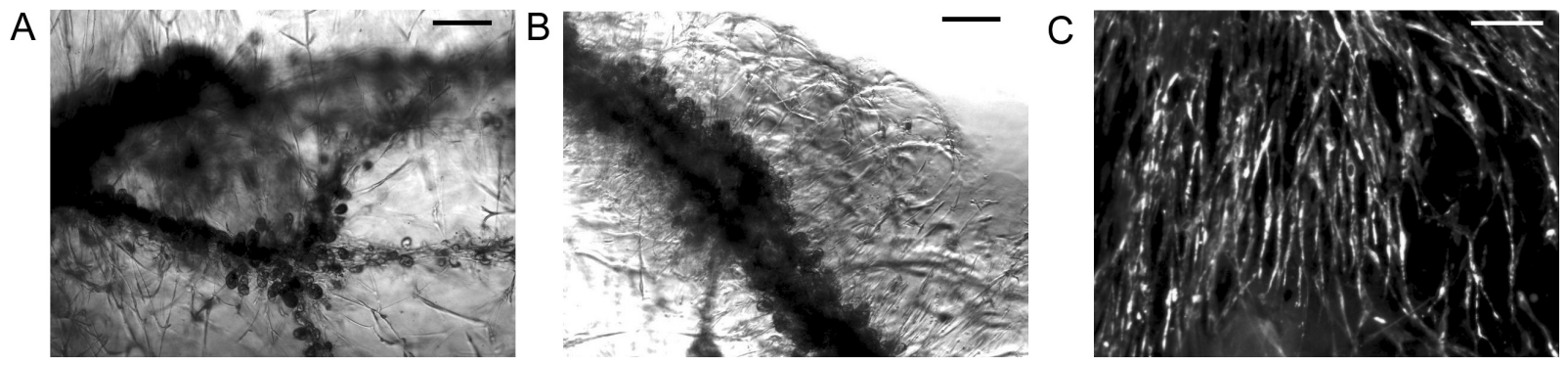

FIG. 7. Fibroblast capsule outgrowth during long-term culture. (A) MVEC-hepatocyte co-culture at day 14. Hepatocytes clearly decorate endothelial structures. Fibroblast-like cells emerge from the MVEC network both in isolation and co-culture. Bar $=200$ $\mu \mathrm{m}$. (B) An outgrowth of fibroblast-like cells from the structures invades the gel, creating a mat on which the structures sit. A clear front of growing cells can be seen by day 14. Bar $=200 \mu \mathrm{m}(\mathbf{C})$ Vimentin staining in fibroblast-like cells growing out from MVEC-hepatocyte co-culture at day 6. Bar $=100 \mu \mathrm{m}$. 

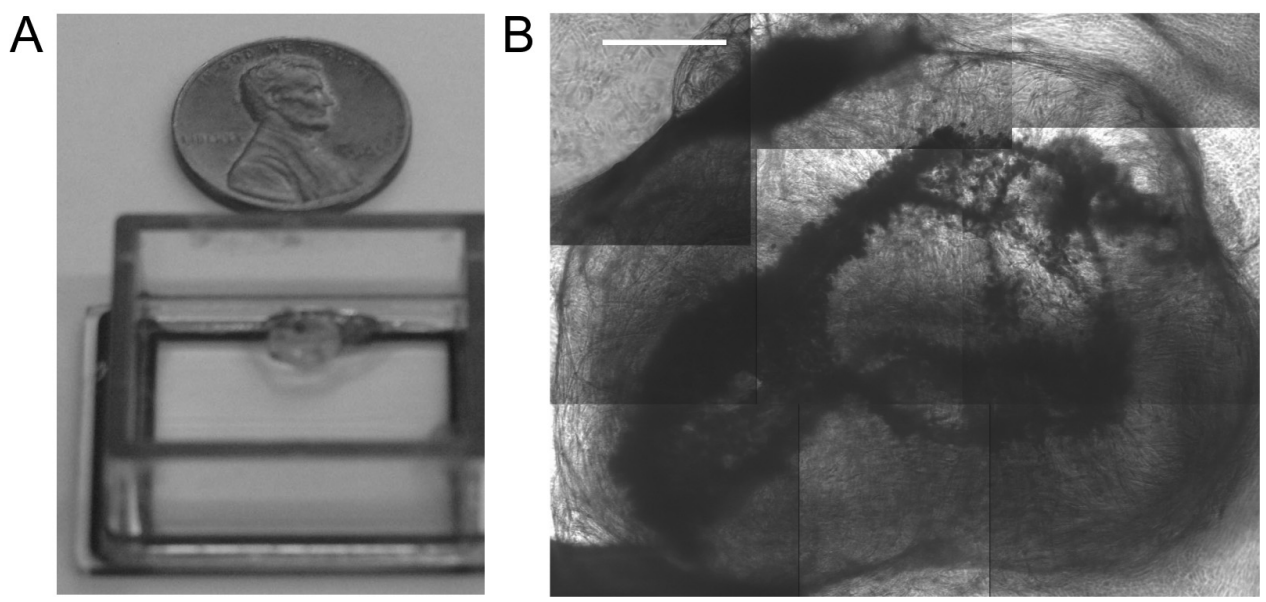

FIG. 8. Liver-like organoid formation. (A) An image of the organoid in tissue culture dish at day 28. Note that the gel has been totally degraded and cannot be seen. The structure is now several millimeters high and is free floating. (B) MVEC-hepatocyte sinusoid-like structures inside a fibroblastic capsule after a month in culture. At this stage, the capsule is free-floating in the media. Bar $=1 \mathrm{~mm}$.

gin (Fig. 7C). Similar outgrowth was present in single cultures of MVEC but not in single cultures of hepatocytes, suggesting that the fibroblast-like cells co-purified with the MVEC. Our findings are consistent with previous studies documenting MVEC impurities. ${ }^{23,32-34}$

To examine whether contaminating fibroblasts were allowing for the stability of MVEC sinusoid-like structures, HUVEC were cultured with NHDF $\left(10,000 \mathrm{cell} / \mathrm{cm}^{2}\right)$ and hepatocytes. HUVEC-NHDF-hepatocyte tricultures showed remarkable similarities to MVEC-hepatocyte cocultures (Fig. 3), suggesting that fibroblasts allowed for endothelial stability in culture. Preservation of endothelial vascular structures by fibroblasts has been previously reported. ${ }^{35}$ Albumin secretion in HUVEC-NHDF-hepatocyte tricultures remained stable for more than 44 days in culture $(23 \mu \mathrm{g} / \mathrm{mL} / 48 \mathrm{~h})$ while single cultures of hepatocytes on Matrigel lost this liver-specific function by day 44 in vitro $(3 \mu \mathrm{g} / \mathrm{mL} / 48 \mathrm{~h})$.

Regardless of fibroblast origin, after 4 weeks a fibroblastic capsule surrounded the MVEC or HUVEC-NHDF-derived sinusoid-like structures, the gel was completely degraded (Fig. 8A) and the capsule detached (Fig. 8B).

\section{Maintenance of hepatocyte function}

Aggregation of hepatocytes around vascular tubes preserved hepatocyte function during short-term culture. $\mathrm{Cy}$ tochrome P450 activity ( $n=3$, Fig. 9A and B), and albumin secretion were maintained $(n=3$, Fig. 6$)$ at levels comparable to that of hepatic spheroids on Matrigel during short-term culture.

After a month of culture, hepatocyte spheroids began to collapse, leading to loss of cytochrome P450 gene expression (Fig. 9E and F) and activity assessed by PROD assay (Fig. 9C). However, hepatocyte-endothelial co-cultures maintained hepatic cytochrome $\mathrm{P} 450$ gene expression (Fig. 9E and F) and activity (Fig. 9D) at high levels even after 40 days of culture in vitro. Similar results were seen for albumin secretion (Fig. 6C and F). In addition, hepatocyte-MVEC cultures maintained HNF-1, HNF-3 $\beta$, and HNF-4 expression, while hepatocytes in single culture rapidly lost transcription factor expression within 14 days (data not shown).

\section{Transmission electron microscopy analysis of organoid structure}

Images obtained by transmission electron microscopy showed that the sinusoid structures were composed of cells with differentiated hepatocyte morphology including abundant glycogen granules, extensive rough endoplasmic reticulum, mitochondria, and bile canaliculi between the hepatocytes with mature junctional complexes (Fig. 10A). Endothelial cells formed tubes decorated by hepatocytes, and displayed numerous cytoplasmic holes suggesting the beginning of fenestrations (Fig. 10B). Microvilli extended along the seams between hepatocytes, as well as along the bile canalicular spaces (Fig. 10C).

Transmission electron microscopy images of the encapsulated hepatocyte-endothelial structures after 3 months revealed several long endothelial vessels, and neighboring hepatocyte plate-like structures (Fig. 11A and B). Ultrastructurally, the hepatocytes at the capsule periphery appeared well preserved after 3 months of in vitro culture, whereas those in the structure interior had enlarged peroxisomes. Because the organoid was several millimeters in diameter, cells in the interior were likely deprived of oxygen, ${ }^{3}$ which may explain their stressed appearance, demonstrating the need to align and perfuse 
A
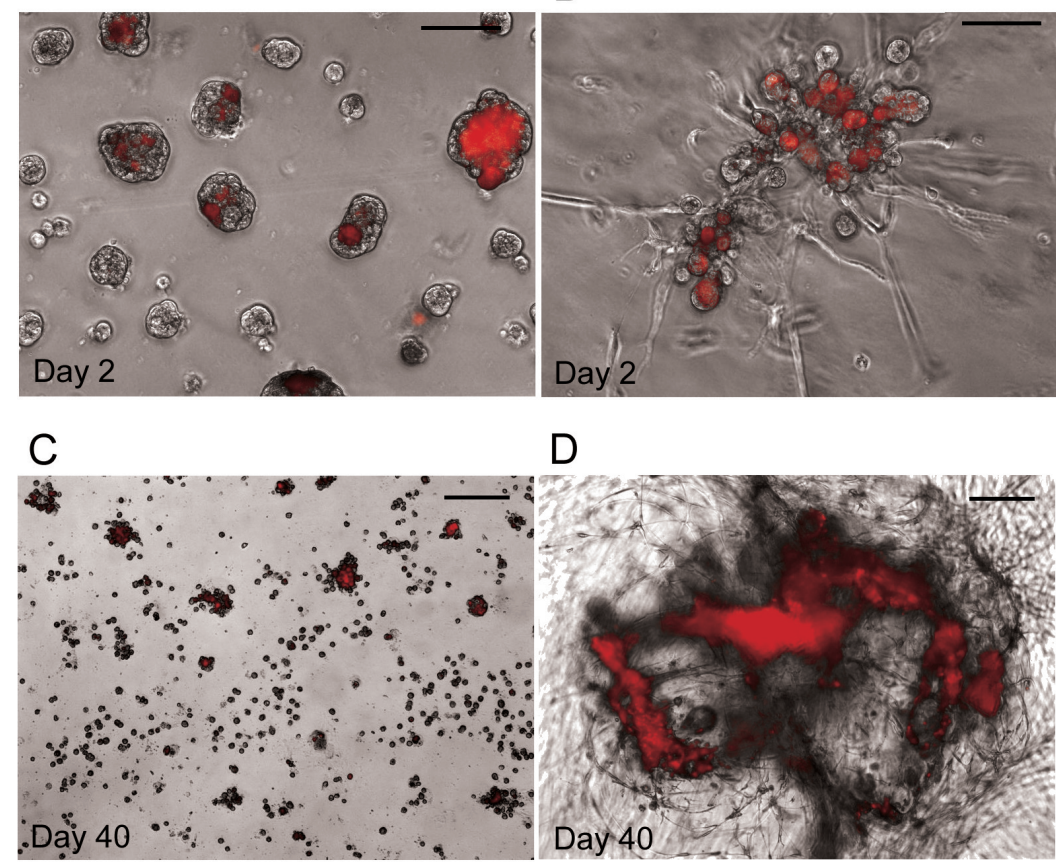

B
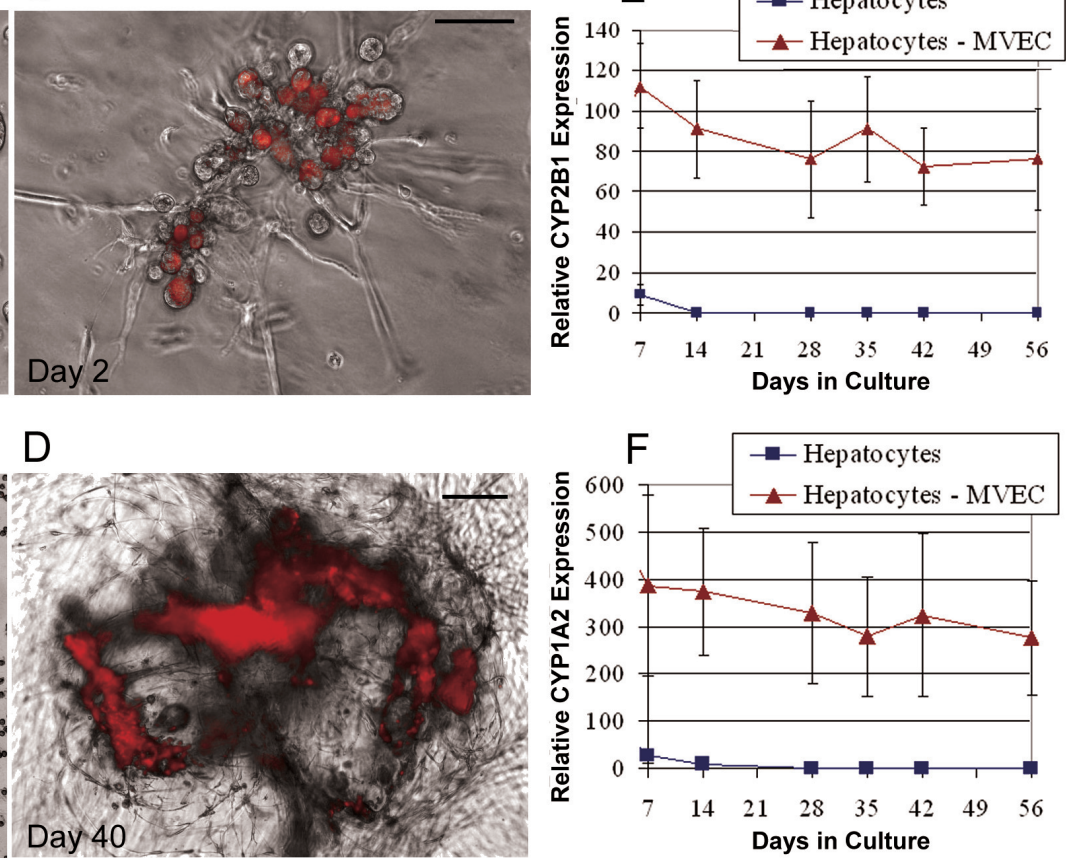

FIG. 9. Cytochrome P450 expression and activity during long-term co-cultures. (A-D) Resorufin accumulation after cleavage of pentoxyresorufin by cytochrome P450 during short- (2 days) and long-term (40 days) culture. (A) Hepatocyte spheroids formed in high density on Matrigel at day 2. Cytochrome P450 activity is present in hepatocyte spheroids, whereas it is virtually undetectable in single hepatocytes and small clusters on Matrigel. (B) Hepatocyte-MVEC co-cultures at day 2. Cytochrome P450 activity is present in hepatocytes that are closely associated with the vascular tubes, while it is virtually undetectable in hepatocytes that are at the structure periphery. Bar $=100 \mu \mathrm{m}(\mathrm{A}, \mathrm{B})$. (C) The remnants of hepatocyte spheroids after 40 days of culture. Very little cytochrome P450 activity can be seen; many hepatocytes are floating dead or dying at this stage. (D) Cultures of HUVEC-NHDF-hepatocytes after 40 days of culture. The hepatic organoid exhibits strong cytochrome P450 activity even after 40 days in culture. Bar $=200 \mu \mathrm{m}(\mathrm{C}, \mathrm{D})$. (E, F) Quantitative reverse transcriptase (RT)-PCR confirms that hepatocyte-MVEC coculture maintains cytochrome P450 CYP2B1, and CYP1A2 expression during long-term culture while a pure hepatocyte culture loses cytochrome P450 expression in 7-14 days on Matrigel. Controls lacking RT were negative for all samples and are not shown. Values are means and standard deviations of three experiments.

the vascular structures during long-term culture with either an oxygen carrying medium or blood.

\section{DISCUSSION}

A growing body of evidence suggests that interaction between endothelium and surrounding tissue plays an intimate role in tissue differentiation, maintenance, and organization. ${ }^{36,37}$ Previous in vivo gene disruption ${ }^{38}$ and in vitro tissue explant studies ${ }^{4}$ showed that HGF is critical for the late stages of liver organogenesis. However, little is known about the molecular mechanisms controlling hepatocyte migration and liver sinusoid organization, ${ }^{39}$ and no studies have addressed the precise role of nascent vascular structures in directing liver morphogenesis. Our studies show that sinusoid-like structures arise in vitro from the interaction between mature hepatocytes and endothelial vascular structures. Contrary to traditional wis- dom, it is the hepatocytes that migrate toward and adhere to endothelial vascular structures in our system, rather than endothelial vascularization of hepatic aggregates. This endothelial-hepatocyte interaction is mediated in part by HGF. Additional work is required to detect whether similar interactions occur in vivo.

Our sinusoid-like structures retained cytochrome P450 activity past day 40 and albumin secretion past day 54 in vitro, when stabilized by fibroblasts/pericytes. Hepatocyte spheroids cultured under the same conditions fail after 28 days in culture. The stabilization of hepatocyte function by fibroblasts has been previously demonstrated, ${ }^{1}$ but the actual organization of endothelial vascular structures coated by hepatocytes is unique to our system. The presence of endothelial vascular structures in our system (Fig. 11A) suggests that similar liver organoid cultures could be perfused, enabling the engineering of liver tissue beyond the oxygen diffusion limit $(\sim 200 \mu \mathrm{m})$. It remains to be seen whether mature ves- 

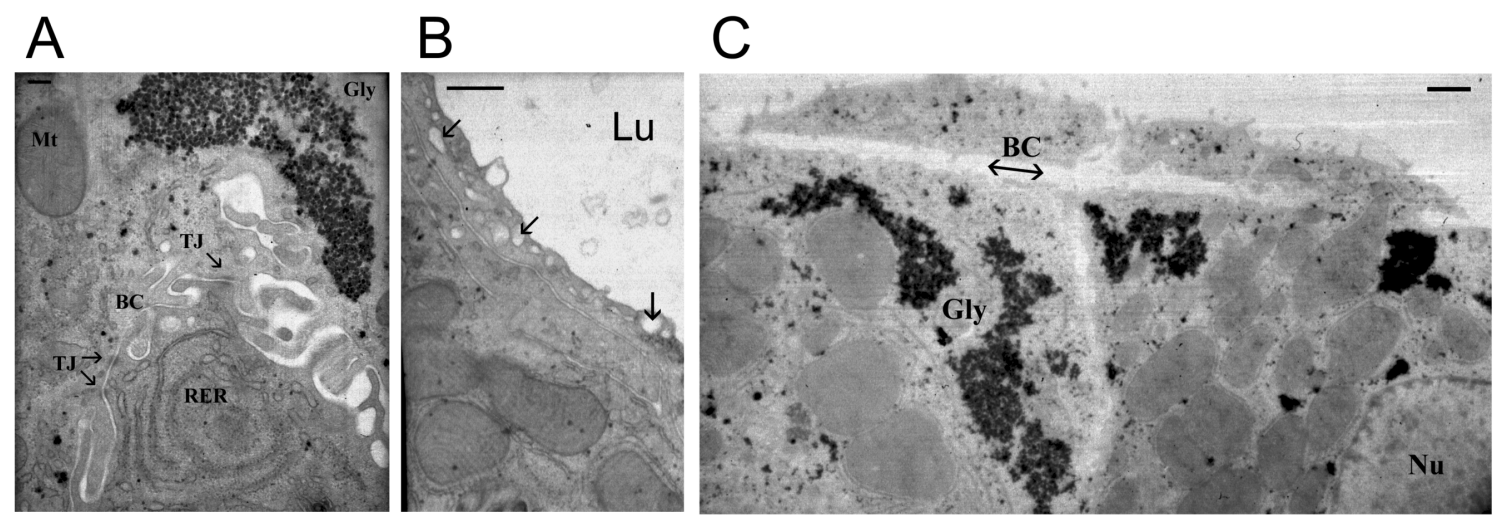

FIG. 10. Ultrastructural analysis by electron microscopy of hepatocyte-MVEC co-culture at day 6. (A) A high-power micrograph of the association between two hepatocytes. Abundant microvilli, extensive rough endoplasmic reticulum (RER), mitochondria (Mt), and glycogen granules can be seen (Gly). Tight junctions (TJ) delimit a small bile canalicular space (BC) between the two cells. Bar $=200 \mathrm{~nm}$. (B) A hepatocyte associated with an endothelial lumen (Lu), membrane-coated holes might characterize fenestrae differentiation (arrows). Bar $=500 \mathrm{~nm}$. (C) A low-power image of two cells and the extensive bile canaliculi network between them. Abundant mitochondria, glycogen granules (Gly), and a round hepatic nucleus (Nu) mark differentiated hepatocytes. Bar $=1 \mu \mathrm{m}$.

sels that can retain solutes can be established in culture. However, our results suggest that endothelial vessels might provide more than the necessary oxygen to the tissue, but also a backbone for liver organization both in vitro and in vivo.

This hypothesis is supported by in vivo observations. In Prox $1^{-1-}$ mouse embryos, the liver forms, although hepatocytes are abnormally absent, suggesting that the nonparenchymal cells of the liver provide considerable structural information. ${ }^{39}$ Similar results have been found in flk- $1^{-1-}$ embryos. ${ }^{4}$ Our in vitro results support these observations and suggest a structural role for nascent en- dothelial vascular structures during liver organogenesis, or regeneration. ${ }^{11}$

In our study, we demonstrate that sinusoid-like structures can be created in vitro using isolated endothelial cells, fibroblasts, and hepatocytes. Early in creation of these structures, HGF mediates migration of hepatocytes toward the endothelial vascular structures. Over a period of 3 weeks, a fibroblast capsule encapsulates the whole structure and it detaches. We have demonstrated that, unlike other systems where hepatocytes lose liver-specific function in 7 to 28 days, hepatocytes in our culture system maintain albumin secretion, and cytochrome P450
A

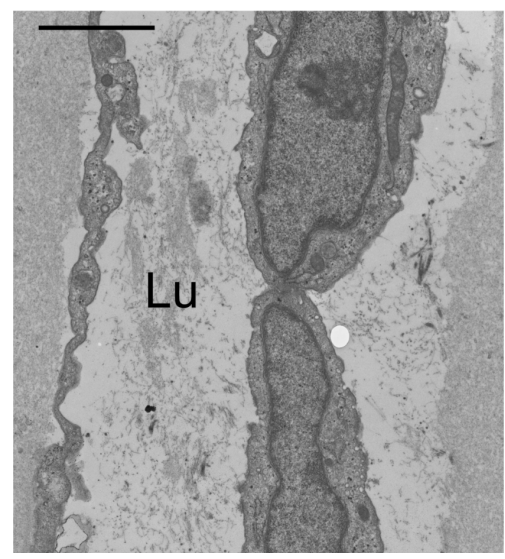

B

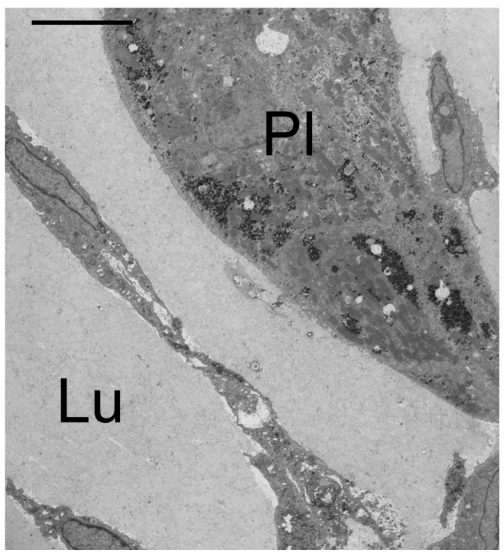

C

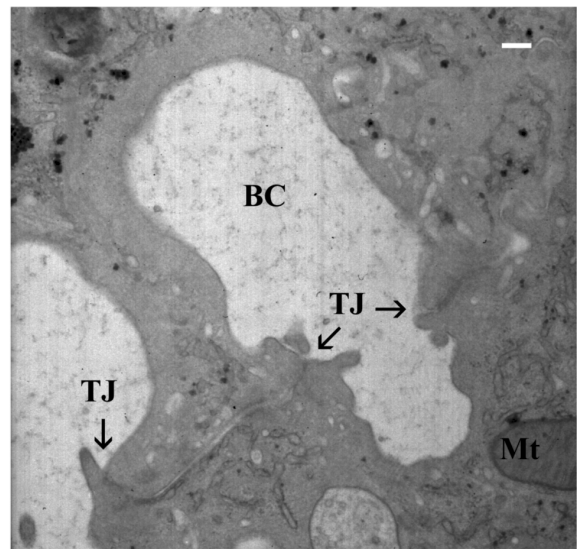

FIG. 11. Ultrastructural analysis by electron microscopy of encapsulated hepatocyte-MVEC organoid capsule after 3 months of culture. (A) Long endothelial vessels stretch from the capsule periphery. Lu, endothelial lumen. Bar $=2 \mu \mathrm{m}$. (B) Hepatocyte plate $(\mathrm{Pl})$, set between endothelial vessels $(\mathrm{Lu})$. Hepatocytes appear well preserved after more than 2.5 months in vitro. Bar $=$ $10 \mu \mathrm{m}$. (C) A bile canaliculi channel (BC) between two hepatocytes. Extensive tight junctional areas (TJ) clearly delimit the channel. Mt, mitochondria. $\mathrm{Bar}=200 \mathrm{~nm}$. 
activity, for up to 54 days in vitro. We therefore suggest that this system and the resulting sinusoid-like structures may serve as a unique in vitro model of the liver for tissue-engineering applications. ${ }^{40}$ The culture conditions should facilitate future efforts to reconstruct and maintain human liver tissue in vitro. The availability of human foreskin MVECs and the small number of human hepatocytes needed, coupled with advances in stem cell biology, make our co-culture system suitable for the study of human liver fibrosis, cirrhosis, ischemia/reperfusion injury, and toxicity. We envision cultures of perfused liver sinusoids suitable for the study of the hepatic microcirculation in disease and the high-throughput screening of drugs in the pharmaceutical industry.

\section{ACKNOWLEDGMENTS}

We thank R. P. Hebbel and J. Nguyen for endothelial cell isolation and culture; S. J. Solis and R. A. Narayanan for hepatocyte harvest; L. K. Hansen for important discussion; M. A. Sanders, K. A. Josephsen, C. D. Frethem, and S. Johnson for electron microscopy support; and J. J. Wilhelm for dot-blots analysis. Supported by NSF Biophotonics Initiative (BES-0119481), NIH grant (U19DK-61224), NIH grant (ROI-HL-71228), the Defense Advanced Research Projects Agency (Tissue-Based Biosensors Program), the MRSEC Program of the National Science Foundation under Award Number DMR0212302, a grant from the $\alpha_{1}$ Antitrypsin Foundation, the University of Minnesota Doctoral Dissertation Fund, and the Biomedical Engineering Institute.

\section{REFERENCES}

1. Bhatia, S.N., Balis, U.J., Yarmush, M.L., and Toner, M. Effect of cell-cell interactions in preservation of cellular phenotype: cocultivation of hepatocytes and non parenchymal cells. FASEB J. 13, 1883, 1999.

2. Fisher, R.L., Ulreich, J.B., Nakazato, P.Z., and Brendel, K. Histological and biochemical evaluation of precision-cut liver slices. Toxicol. Methods 11, 59, 2001.

3. Griffith, L.G., and Naughton, G. Tissue engineering-Current challenges and expanding opportunities. Science $\mathbf{2 9 5}$, 1009, 2002.

4. Matsumoto, K., Yoshitomi, H., Rossant, J., and Zaret, K.S. Liver organogenesis promoted by endothelial cells prior to vascular function. Science 297, 559, 2001.

5. Michalopoulos, G.K., and DeFrances, M.C. Liver regeneration. Science 276, 60, 1997.

6. Fondevila, C., Busuttil, R.W., and Kupiec-Weglinski, J.W. Hepatic ischemia/reperfusion injury-a fresh look. Exp. Mol. Pathol. 74, 86, 2003.

7. Grattagliano, I., Portincasa, P., Palmieri, V.O., and Palasciano, G. Overview on the mechanisms of drug-induced liver cell death. Ann. Hepatol. 1, 162, 2002.
8. Neubauer, K., Saile, B., and Ramadori, G. Liver fibrosis and altered matrix synthesis. Can. J. Gastroenterol. 15, 187, 2001.

9. Gardner, J.P., Durso, R.J., Arrigale, R.R., Donovan, G.P., Maddon, P.J., Dragic, T., et al. L-SIGN (CD 209L) is a liver-specific capture receptor for hepatitis $\mathrm{C}$ virus. PNAS 100, 4498, 2003.

10. Jung, J., Zheng, M., Goldfarb, M., and Zaret, K.S. Initiation of mammalian liver development from endoderm by fibroblast growth factors. Science 284, 1998, 1999.

11. Stolz, D.B., Ross, M.A., Salem, H.M., Mars, W.M., Michalopoulos, G.K., and Enomoto, K. Cationic colloidal silica membrane perturbation as a means of examining changes at the sinusoidal surface during liver regeneration. Am. J. Pathol. 155, 1487, 1999.

12. Mitaka, T., Sato, F., Mizuguchi, T., Yokono, T., and Mochizuki, Y. Reconstruction of hepatic organoid by rat small hepatocytes and hepatic nonparenchymal cells. Hepatology 29, 111, 1999.

13. Mitaka, T. Reconstruction of hepatic organoid by hepatic stem cells. J Hepatobiliary Pancreat. Surg. 9, 697, 2002.

14. Michalopoulos, G.K., Bowen, W.C., Zajac, V.F., Stolz, D.B., Watkins, S., Kostrubsky, V., et al. Morphogenetic events in mixed cultures of rat hepatocytes and nonparenchymal cells maintained in biological matrices in the presence of hepatocyte growth factor and epidermal growth factor. Hepatology 29, 90, 1999.

15. Michalopoulos, G.K., Bowen, W.C., Mule, K., and Stolz, D.B. Histological organization in hepatocyte organoid cultures. Am. J. Pathol. 159, 1877, 2001.

16. Bhatia, S.N., Yarmush, M.L., and Toner, M. Controlling cell interactions by micropatterning in co-cultures: Hepatocytes and $3 \mathrm{~T} 3$ fibroblasts. J. Biomed. Materials Res. 34, $189,1997$.

17. Harimoto, M., Yamato, M., Hirose, M., Takahashi, C., Isoi, Y., Kikuchi, A, et al. Novel approach for achieving double-layered cell sheets co-culture: overlaying endothelial cell sheets onto monolayer hepatocytes utilizing temperature-responsive culture dishes. J. Biomed. Material Res. 62, 464, 2002.

18. Ballet, F. Hepatotoxicity in drug development: detection, significance and solutions. J. Hepatol. 26, 26, 1997.

19. Xu, B., Broome, U., Uzunel, M., Nava, S., Ge, X., Kumagai-Braesch, M., et al. Capillarization of hepatic sinusoid by liver endothelial cell-reactive autoantibodies in patients with cirrhosis and chronic hepatitis. Am. J. Pathol. 163, 1275, 2003.

20. Kohn, E.C., Alessandro R., Spoonster, J., Wersto, R.P., and Liotta, L.A. Angiogenesis: Role of calcium-mediated signal transduction. PNAS 92, 1307, 1995.

21. Seglen, P.O. Preparation of isolated rat liver cells. Methods Cell Biol. 13, 29, 1976.

22. Zhang, B., Borderie, D., Sogni, P., Soubrane, O., Houssin, D., and Calmus, Y. NO-mediated vasodilation in the rat liver, Role of hepatocytes and liver endothelial cells. J. Hepatol. 26, 1348, 1997.

23. Gupta, K., Ramakrishnan, S., Browne, P.V., Solovey, A., and Hebbel, R.P. A novel technique for culture of human dermal microvascular endothelial cells under either serumfree or serum-supplemented conditions: isolation by pan- 
ning and stimulation with vascular endothelial growth factor. Exp. Cell Res. 230, 244, 1997.

24. Matsumoto, K., Okazaki, H., and Nakamura, T. Novel function of prostaglandins as inducers of gene expression of HGF and putative mediators of tissue regeneration. J. Biochemistry (Tokyo) 117, 458, 1995.

25. Burke, M.D., Thompson, S., Weaver, R.J., Wolf, C.R., and Mayer, R.T. Cytochrome P450 specificities of alkoxyresorufin O-dealkylation in human and rat liver. Biochem. Pharmacol. 48, 923, 1994.

26. Cretton, E.M., and Sommadossi, J.P. Modulation of 3'azido-3'-deoxythymidine catabolism by probenecid and acetaminophen in freshly isolated rat hepatocytes. Biochem. Pharmacol. 42, 1475, 1991.

27. Kubota, Y., Kleinman, H.K., Martin, G.R., and Lawley, T.J. Role of laminin and basement membrane in the morphological differentiation of human endothelial cells into capillary-like structures. J. Cell Biol. 107, 1589, 1988.

28. Moghe, P.V., Coger, R.N., Toner, M., and Yarmush, M.L. Cell-cell interactions are essential for maintenance of hepatocyte function in collagen gel but not on Matrigel. Biotechnol. Bioengineering 56, 706, 1997.

29. Tachibana, K., Hirota, S., Iizasa, H., Yoshida, H., Kawabata, K., Kataoka, Y., et al. The chemokine receptor CXCR4 is essential for vascularization of the gastrointestinal tract. Nature 393, 591, 1998.

30. Kollet, O., Shivtiel, S., Chen, Y.-Q., Suriawinata, J., Thung, S.N., Dabeva, M.D., et al. HGF, SDF-1, and MMP-9 are involved in stress-induced human CD34+ stem cell recruitment to the liver. J. Clin. Invest. 112, 160, 2003.

31. Hatch, H.M., Zheng, D., Jorgensen, M.L., and Petersen, B.E. SDF-1alpha/CXCR4: a mechanism for hepatic oval cell activation and bone marrow stem cell recruitment to the injured liver of rats. Cloning Stem Cells 4, 339, 2002.
32. Cha, M.S., Rah, D.K., and Lee, K.H. Isolation and pure culture of microvascular endothelial cells from the fetal skin. Yonsei Med. J. 37, 186, 1996.

33. Hewett, P.W., and Murray, J.C. Isolation, culture and properties of microvessel endothelium from human breast adipose tissue. In: Bicknell, R., ed. Endothelial Cell Culture. Cambridge: Cambridge University Press, 1996, pp. 55-76.

34. Ruszczak, Z. Human skin microvascular endothelial cells. In: Bicknell, R., ed. Endothelial Cell Culture. Cambridge: Cambridge University Press, 1996, pp. 77-89.

35. Koike, N., Fukumura, D., Gralla, O., Au, P., Schechner, J.S., and Jain, R.K. Creation of long-lasting blood vessels. Nature 428, 138, 2004.

36. Bahary, N., and Zon, L.I. Endothelium-Chicken soup for the endoderm. Science 294, 530, 2001.

37. Cleaver, O., and Melton, D.A. Endothelial signaling during development. Nature Medicine 9, 661, 2003.

38. Schmidt, C., Bladt, F., Goedecke, S., Brinkmann, V., Zschiesche, W., Sharpe, M., et al. Scatter factor/hepatocyte growth factor is essential for liver development. Nature 373, 699, 1995.

39. Sosa-Pineda, B., Wigle, J.T., and Oliver, G. Hepatocyte migration during liver development requires Prox1. Nature Genetics 25, 254, 2000.

40. Strain, A.J. Ex vivo liver cell morphogenesis: One step nearer to the bioartificial liver? Hepatology 29, 288, 1999.

Address reprint requests to:

David J. Odde

Department of Biomedical Engineering University of Minnesota 7-105 BSBE

312 Church Street Minneapolis, MN 55455

E-mail: oddex002@umn.edu 